\title{
ANÁLISE DE FATORES CLÍNICOS E HISTOPATOLÓGICOS EM METÁSTASES HEPÁTICAS DE ADENOCARCINOMA COLORRETAL
}

\section{Analysis of clinical and histopathological factors in adenocarcinoma colorectal cancer liver metastases}

\author{
Jefferson Cláudio MURAD ${ }^{1}$, Ulysses RIBEIRO-Jr'1 , Carlos Eduardo CORBETT ${ }^{2}$, Viviane RAWET ${ }^{2}$, Venâncio A. \\ FERREIRA $^{2}$, Vincenzo PUGLIESE ${ }^{1}$, E. MASSAD ${ }^{2}$, William Abraão SAAD ${ }^{1}$, \\ Ivan CECCONELLO' ${ }^{1}$, Angelita HABR-GAMA ${ }^{1}$, Joaquim GAMA-RODRIGUES ${ }^{1}$
}

ABCDDV/567

Murad JC, Ribeiro Jr. U, Corbett CE, Rawet V, Ferreira VA, Puglise V, Massad E, Saad WA, Cecconello I, Habr-Gama A, Gama-Rodrigues J. Análise de fatores clínicos e histopatológicos em metástases hepáticas de adenocarcinoma colorretal. ABCD Arq Bras Cir Dig 2007;20(4):241-4

RESUMO - Racional - O câncer colorretal inclui-se entre as primeiras neoplasias malignas mais freqüentes no mundo e causa de morte entre os diversos tipos de câncer; ultrapassado somente pelo câncer de pulmão. Freqüentemente ocorrem metástases e o agravamento da doença levando à morte Objetivo - Avaliar se a ressecção cirúrgica radical das metástases hepáticas com margem de segurança superior a 10 mm promove maiores índices de sobrevivência e quais os fatores que podem auxiliar no prognóstico. Métodos - Análise retrospectiva de 49 pacientes portadores de metástase hepática de adenocarcinoma colorretal, sem evidência de concomitância em outros órgãos e submetidos a tratamento cirúrgico. Os indicadores epidemiológicos foram: idade, gênero, tamanho da metástase hepática e ou da maior lesão, número de nódulos regionais ressecados e comprometidos, margem de ressecção livre de neoplasia. Os sobreviventes foram convocados e avaliados clinicamente, por meio de exames laboratoriais e estudos radiológicos com finalidade de determinar a evolução da doença. Os critérios de exclusão foram falta de comprovação histológica da metástase hepática e com evidência de neoplasia em outros órgãos além do intestino grosso e do fígado, na época do tratamento cirúrgico inicial e da metástase hepática. Resultados - A casuística consistiu de 24 pacientes do gênero feminino e 25 do masculino.A média e o desvio-padrão das idades foi de 55,9 + 11,9 anos com mediana de 56 anos, Foram realizadas 15 hepatectomias direitas regradas e 11 esquerdas; 13 segmentectomias direitas e esquerdas; 9 nodulectomias e 1 biópsia. Adicionalmente efetuaram-se 2 alcoolizações, 4 quimioembolizações, 1 termoablação, 1 bloqueio portal seletivo com posterior hepatectomia direita e termoablação de lesões no segmentos III e IV. O peso do fígado foi igual a 555,71 + 261,96 g e mediana de $600 \mathrm{~g}$. O número mediano de nódulos ressecados foi de 2 . O tamanho médio da lesão foi de 4,45+2,8. A margem cirúrgica maior que $10 \mathrm{~mm}$ foi observada em 32 casos. O valor do CEA antes da operação de 68,13+105,65 ng/ml e mediana de 22,2 ng/ml. Obito ocorreu em 22 casos $(44,89 \%)$. O tipo histológico predominante foi o adenocarcinoma tubular moderadamente diferenciado em $65,96 \%, 17,02 \%$ pouco e $17,02 \%$ bem diferenciado. Fatores como o tipo histológico indiferenciado, menor infiltrado inflamatório peritumoral, maior reação desmoplásica e inexistência de cápsula circunscrevendo o tumor parecem compor fatores de pior prognóstico, embora não tenham sido capazes de isoladamente serem significantes Observou-se associação significante entre o nível sérico abaixo de $7 \mathrm{ng} / \mathrm{ml}$ de CEA e o sincronismo da metástase hepática. Conclusões - A ressecção cirúrgica radical das metástases hepáticas com margem de segurança superior a $10 \mathrm{~mm}$ promoveram maior sobrevida; os níveis séricos elevados de CEA associaram-se à recidiva tumoral das metástases e pior evolução clínica; 3. tipo histológico indiferenciado, menor infiltrado inflamatório peritumoral, maior reação desmoplásica, inexistência de cápsula circunscrevendo o tumor sugerem pior prognóstico.

DESCRITORES -

\section{INTRODUÇÃO}

O câncer colorretal situa-se entre as neoplasias malignas mais freqüentes do mundo e das mais comuns causa de morte entre os diversos tipos de câncer, sendo ultrapassado somente pelo câncer de pulmão ${ }^{1}$.

Nas metástases do fígado, é consenso na literatura que lesões maiores que 3 ou $4 \mathrm{~cm}$ devam ser tratadas com ressecção hepática regrada clássica, preferencialmente. Quando ela é maior que $5 \mathrm{~cm}$, ou quando há mais que 3 lesões neoplásicas no fígado, nota-se piora do prognóstico ${ }^{2}$.

A margem cirúrgica livre da lesão hepática é considerada fator prognóstico negativo quando menor que 10

Trabalho realizado na Clinica Cirúrgica II Hospital das Clínicas da Faculdade de Medicina da Universidade de São Paulo ${ }^{1}$ e Departamento de Patologia da Faculdade de Medicina da Universidade de São Paulo², São Paulo, SP, Brasil.

Endereço para correspondência: $\mathrm{mm}$, e como critério de radicalidade cirúrgica².

$\mathrm{Na}$ evolução e prognóstico, o controle do CEA é bom marcador na determinação de recidiva e metástases, além de que quanto alto ou em elevação é fator de mau prognóstico.

Além disso, os achados histológicos do tumor primário no cólon, que quando mostram necrose, infiltrado inflamatório, desmoplasia e ocorrência de cápsula, indicam comprometimento na evolução clínica desses pacientes ${ }^{4}$. A reação desmoplásica é processo que envolve produção excessiva de tecido conjuntivo junto ao tumor, sendo pouco estudado até o momento ${ }^{4}$. Rawet ${ }^{4}$ detectou no sítio primário, em adenocarcinoma colorretal, que índices moderados ou intensos do infiltrado inflamatório, apresentavam sobrevida significantemente maior. No crescimento tumoral estão envolvidos fatores ligados ao estroma do tumor onde a célula se desenvolve, podendo atuar nas propriedades invasivas e metastáticas das células 
malignas. O tipo histológico predominante da metástase hepática do câncer colorretal é o adenocarcinoma tubular moderadamente diferenciado.

A formação de cápsula peritumoral, formada a partir de macrófagos, poderia representar barreira à progressão tumoral, embora essa não seja completa ou eficiente na maioria dos casos.

Assim, este estudo tem por objetivos avaliar se a ressecção cirúrgica radical das metástases hepáticas com margem de segurança superior a $10 \mathrm{~mm}$ aumenta a sobrevida e quais os fatores que podem auxiliar no prognóstico.

\section{MÉTODOS}

Foram estudados 49 pacientes portadores de metástase hepática de adenocarcinoma colorretal, sem evidência de concomitância em outros órgãos, e submetidos a tratamento cirúrgico no período de 1992 a 2002. Analisaram-se retrospectivamente os dados clínicos desses pacientes para verificação da idade, gênero, peso e altura, tamanho da metástase hepática e ou da maior lesão, número de nódulos ressecados e comprometidos e a margem de ressecção livre de neoplasia. Adicionalmente verificou-se a realização de tratamentos complementares ao cirúrgico, o obituário com o procedimento e evolução, e a correlação existente entre fatores clínicos e histopatológicos. Quando ressecado, o peso médio do lobo hepático foi medido atribuindo-se valor igual a 0 quando o tecido correspondia até $0,4 \%$ do peso corpóreo; de $0,4 \%$ a $0,8 \%$ valor igual a 1 ; acima de $0,8 \%$, igual a 2 .

Os pacientes sobreviventes foram convocados e avaliados clinicamente, por meio do antígeno cárcino-embrionário (CEA) e estudos radiológicos com finalidade de determinar o estádio de evolução da doença.

Foram excluídos pacientes para os quais não se conseguiu comprovação histológica da metástase hepática e com evidência de neoplasia em outros órgãos além do intestino grosso e do fígado, na época do tratamento cirúrgico inicial e da metástase hepática.

No estudo histológico das lâminas do espécime cirúrgico do fígado coradas com Hematoxilina-eosina, observou-se o tipo tumoral, a presença e intensidade da necrose graduada de 0 a 3 , desmoplasia graduada de 1 a 3 , infiltrado inflamatório graduado de 0 a 3 e a presença ou não de cápsula.

$\mathrm{Na}$ análise estatística utilizou-se o método de KaplanMeyer para curvas de sobrevivência, o teste de log-rank ${ }^{3}$ para análise do efeito de cada variável sobre o intervalo livre de doença e sobrevivência média de tempo em meses, o Qui-quadrado para 2 valores de $\mathrm{P}$ e o teste de correlações de PEARSON e Spearman ${ }^{3}$ para verificar as associações entre os fatores histopatológicos e dados clínicos.

\section{RESULTADOS}

Foram 24 pacientes do gênero feminino e 25 do masculino. A média e o desvio-padrão das idades foi de 55,9 + 11,9 anos com mediana de 56 anos e mínima de 24 e máxima de 77 anos. $O$ peso corpóreo médio foi de $67,9+$
$14 \mathrm{~kg}$ e altura média de $1,63+9,8 \mathrm{~cm}$.

Os procedimentos cirúrgicos utilizados para a retirada dos nódulos foram: hepatectomia direita regrada em 15 pacientes, hepatectomia esquerda regrada em 11; segmentectomias direita e esquerda em 13; nodulectomias em 9 e biópsia em 1 .

Os procedimentos terapêuticos complementares foram: 2 alcoolizações; 4 quimioembolizações; 1 termoablação e 1 bloqueio portal seletivo com posterior hepatectomia direita e termoablação de lesões no segmentos III e IV.

O peso do segmento hepático ressecado oscilou de 45 a $960 \mathrm{~g}$. O peso médio foi igual a $555,71+261,96 \mathrm{~g}$ com mediana de $600 \mathrm{~g}$. O número de nódulos variou sendo a mediana de 2. O tamanho da lesão variou de 0,6 a $11 \mathrm{~cm}$ de diâmetro no seu maior eixo com tamanho médio de $4,45+2,8$.

A margem cirúrgica maior que $10 \mathrm{~mm}$ foi observada em 32 casos. Em 17 ela foi menor que $1 \mathrm{~cm}$. Em 31 casos $(63,2 \%)$ metástase hepática já estava presente no procedimento cirúrgico inicial ou apareceu até 6 meses após. Em 18 casos $(36,8 \%)$ o aparecimento da metástase foi metacrônica, isto é, apareceu após 6 meses da cirurgia colorretal.

O valor do CEA médio pré-operatório foi igual a 68,13 $+105,65 \mathrm{ng} / \mathrm{ml}$ com mediana de $22,2 \mathrm{ng} / \mathrm{ml}$. Ele variou de 0,2 a $400 \mathrm{ng} / \mathrm{ml}$.

O óbito ocorreu em 22 casos (44,89\%). Quando se separou o grupo de pacientes que foram submetidos a tratamento cirúrgico radical $(\mathrm{n}=28)$, e analisou-se a sobrevivência total em 36 meses, o número de óbitos foi igual a $9(32,14 \%)$ e sobrevivência de 19 pacientes $(67,56 \%)$.

O tipo histológico predominante foi o adenocarcinoma tubular moderadamente diferenciado em 31 casos $(65,96$ $\%)$. Oito casos $(17,02 \%)$ foram do tipo pouco diferenciado e outros 8 do tipo bem diferenciado. Em 1 caso, verificouse fibrose da lesão em paciente anteriormente submetido à quimioembolização. Necrose foi observada em 47 casos e ausente em 1. Foi localizada em 16 casos $(34,04 \%)$, moderada em $12(25,53 \%)$ e extensa em 18 (38,29\%).

A reação desmoplásica foi leve em 17 casos (36,18\%), moderada em 24 (51,06\%) e intensa em 6 (12,76\%) (Figura 1).

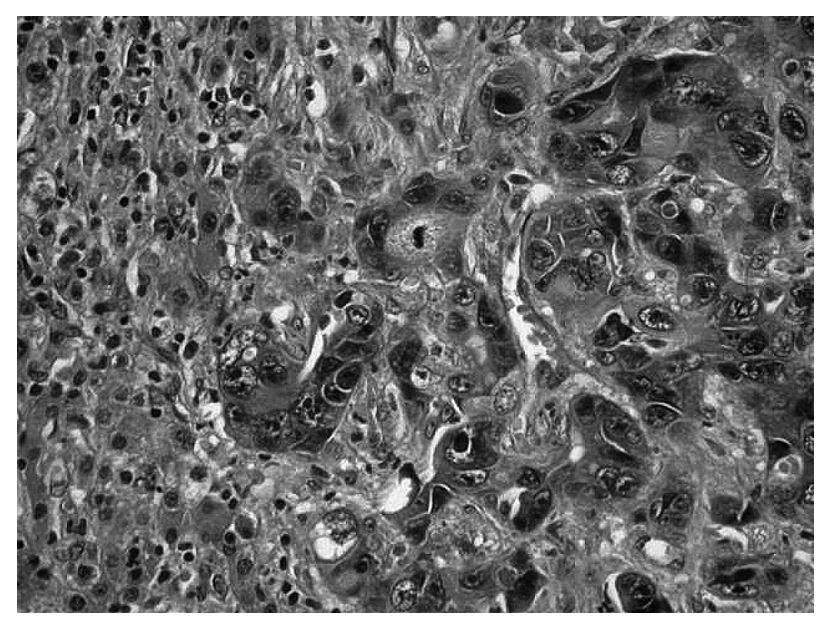

FIGURA 1 - Reação desmoplásica metástase hepática de adenocarcinoma colorretal (Aumento 400x) 
Infiltrado inflamatório foi inexistente em 1 caso (2,27\%); leve em 23 (48,93\%); moderado em 21 (44,68\%) e intenso em $2(04,25 \%)$.

A presença de cápsula ocorreu em 14 casos $(29,78 \%)$ (Figura 2).

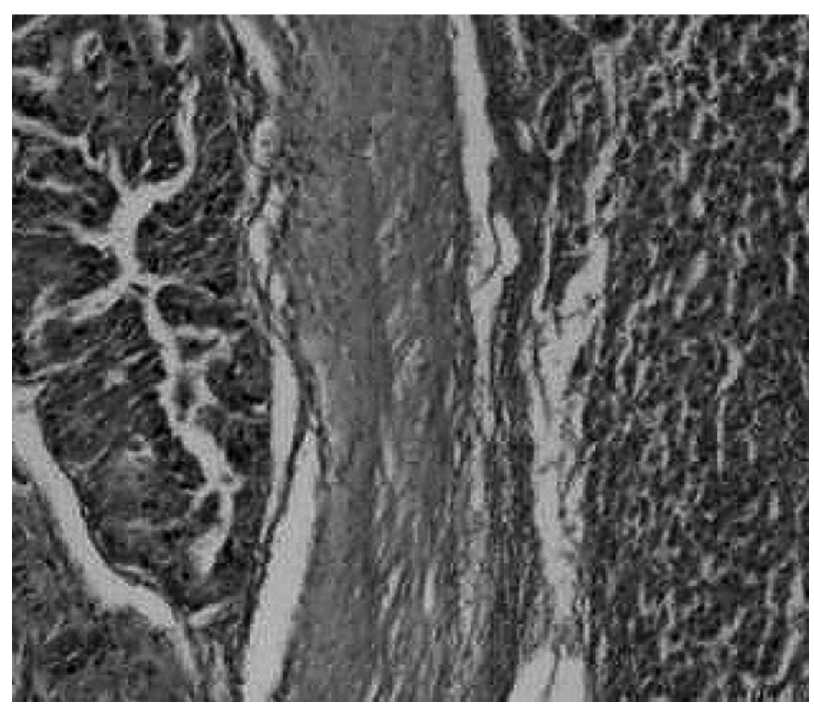

FIGURA 2 - Metástase hepática mostrando em detalhe cápsula peritumoral (Aumento 400x)

A margem cirúrgica maior que $1 \mathrm{~cm}(\mathrm{P}=0,004)$ e o nível de $C E A$ inferior a $7 \mathrm{ng} / \mathrm{ml}(\mathrm{P}=0,04)$ associaram-se a maior sobrevivência acumulada.

Quando se analisou a correlação existente entre fatores clínicos e histopatológicos, observou-se que existiu associação significante inversa entre o tamanho da lesão e a intensidade de infiltrado inflamatório.

Observou-se associação significante entre o nível sérico abaixo de $7 \mathrm{ng} / \mathrm{ml}$ de CEA e o sincronismo da metástase hepática.

Houve tendência de associação significante direta entre o peso maior relativo do tecido hepático ressecado e o tipo histológico pouco diferenciado. Em contrapartida, não notou-se diferença estatística em relação ao tempo de sobrevivência para todo o grupo de pacientes.

\section{DISCUSSÃO}

A margem cirúrgica livre da lesão hepática é considerada fator prognóstico negativo quando menor que $10 \mathrm{~mm}$, e ele é usado como critério de radicalidade cirúrgica ${ }^{2}$, o que também foi aqui demonstrado.

O CEA (antígeno cárcino-embrionário) esteve elevado na maioria dos casos antes da ressecção cirúrgica da metástase hepática e ocorreu evidente elevação dele quando ocorreu evolução da doença e recidiva metastática. Persiste ainda na literatura a concepção de que o CEA é bom marcador de recidiva e metástases e está correlacionado com mau prognóstico. Este fato também pôde ser demonstrado neste estudo.

Rawet ${ }^{4}$ analisou fatores histológicos do tumor primário no cólon em pacientes operados por adenocarcinoma colorretal e mostrou que indicadores histológicos, tais como necrose, infiltrado inflamatório, desmoplasia e ocorrência de cápsula têm importância na evolução clínica e prognóstico desses pacientes.

Na presente casuística, óbito ocorreu em 22 casos do total de 49 pacientes operados pelo menos há 36 meses, o que correspondeu a $44,8 \%$. Não houve variação entre os que se submeteram a tratamento com maior ou menor ressecção hepática.

Quando se analisou a influência do tratamento cirúrgico mais radical em relação à sobrevivência, verificou-se que esse foi fator significante de prognóstico favorável.

Ao se analisar o intervalo livre de doença, para o grupo de pacientes que foi submetido à ressecção observou-se que houve sobrevivência de 70,37\% em 3 anos, e o óbito, nesse grupo estudado ocorreu em 8 casos $(29,63 \%)$. O Quadro 1 mostra a sobrevida referida por vários autores quando trataram as metástases hepáticas e fica claro que o tratamento cirúrgico radical continua sendo de grande importância para o prolongamento da vida do paciente e do intervalo livre de doença.

QUADRO 1 - Sobrevida após ressecção de metástase hepática por adenocarcinoma colorretal

\begin{tabular}{lccc}
\hline \multicolumn{1}{c}{ Autores } & \multicolumn{3}{c}{ Sobrevivência dos pacientes (n) } \\
& $\mathbf{3}$ anos & $\mathbf{5}$ anos & Livre da doença \\
\hline BUTLER et al., 1986 & $50 \%$ & $34 \%$ & 62 \\
EKBERG et al., 1986 & $30 \%$ & $16 \%$ & 72 \\
BRADPIECE et al., 1987 & $44 \%$ & - & 24 \\
NORDLINGER et al., 1987 & $41 \%$ & $25 \%$ & 80 \\
HUGHES et al., 1989 & - & $32 \%$ & 800 \\
SCHLAG et al., 1990 & $40 \%$ & $30 \%$ & 122 \\
ROSEN et al., 1992 & $47 \%$ & $25 \%$ & 280 \\
NAKAMURA et al., 1992 & $45 \%$ & $45 \%$ & 31 \\
GAYOWSKI et al., 1994 & $43 \%$ & $32 \%$ & 204 \\
SCHEELE et al., 1995 & $45 \%$ & $33 \%$ & 434 \\
ROUGIER et al., 1995 & $35 \%$ & $21 \%$ & 123 \\
GAMA-RODRIGUES et al., 1998 & - & $32 \%$ & 32 \\
MURAD et al., 2003 & $70 \%$ & $38 \%$ & 27 \\
\hline
\end{tabular}

\section{CONCLUSÕES}

1. A ressecção cirúrgica radical das metástases hepáticas com margem de segurança superior a $10 \mathrm{~mm}$ promove maior sobrevida; 2. os níveis séricos elevados de CEA associaram-se à recidiva tumoral das metástases e pior evolução clínica; 3. tipo histológico indiferenciado, menor infiltrado inflamatório peritumoral, maior reação desmoplásica, inexistência de cápsula circunscrevendo o tumor sugerem pior prognóstico. 
Murad JC, Ribeiro Jr. U, Corbett CE, Rawet V, Ferreira VA, Puglise V, Massad E, Saad WA, Cecconello I, Habr-Gama A, Gama-Rodrigues J. Analysis of clinical and histopathological factors in adenocarcinoma colorectal cancer liver metastases. ABCD Arq Bras Cir Dig 2007;20(4):241-4.

ABSTRACT - Background - Colorectal cancer belongs to the most frequent malignant neoplasia in the world and responsible for the cause of death among other types of cancer; ranked second behind lung cancer. Metastasis frequently occurs and disease worsening leads to patient death. Aim - To analyze if radical surgical resection for colorectal cancer liver metastases with resection margin greater than 10 mm promotes better survival rates and the factors that might predict prognosis. Methods - Retrospective analysis of 49 patients presenting colorectal adenocarcinoma liver metastases without evidence of concomitant disease and submitted to surgical treatment. Epidemiologic parameters were: age, gender, size of liver metastasis and or the largest lesion, number of regional lymph nodes dissection and involvement, neoplasia-free margin resection. Patients were evaluated clinically, undergoing laboratory exams analysis and imaging studies for disease follow-up. Exclusion criteria were non-histological proof of liver metastasis and evidence of disease in sites other than colon and liver, at the time of surgical treatment and liver metastasis. Results - Casuistic group consisted of 24 female and 25 male patients. Mean and standard deviation for age was 55,9+11,9 years, median of 56 years. Surgical procedures included 15 right hepatectomy and 11 left hepatectomy; 13 right and left segmentectomy; 9 nodulectomy and 1 biopsy. Additionally, 2 alcoholization, 4 chemoembolization, 1 thermoablative therapy, 1 selective portal vein block with later right hepatectomy and thermoablative thereapy on segments III and IV were performed. Liver weighted 555,71 + 261,96 g, median of 600g. Median of lymph nodes resection was 2 . The mean lesion size consisted in 4,45 +2,8. Resection margin greater than $10 \mathrm{~mm}$ was observed in 32 cases. Serum CEA value before surgical procedure was $68,13+105,65 \mathrm{ng} / \mathrm{ml}$, median of $22,2 \mathrm{ng} / \mathrm{ml}$. Death occurred in 22 cases (44,89\%). Predominant histological diagnoses was moderate differentiated tubular adenocarcinoma in $65,96 \%, 17,02 \%$ poorly and $17,02 \%$ well differentiated. Factors such as undifferentiated histological type, less inflammatory peritumor infiltration, greater desmoplastic reaction and the absence of capsule around the tumor seem to reflect worse prognosis, although none of the factors being statistic significantly isolated. Significant association was noticed between CEA serum level under $7 \mathrm{ng} / \mathrm{mg}$ and synchronic hepatic metastases. Conclusion - Radical surgical resection for colorectal cancer liver metastases with a resection margin greater than $10 \mathrm{~mm}$ promotes better survival rates; elevated serum CEA levels were related to recurrence after hepatic resection for metastatic colorectal cancer and worse clinical outcome; undifferentiated histological type, less inflammatory peritumor infiltration, greater desmoplastic reaction and the absence of capsule around the tumor suggested worse prognosis.

HEADINGS - Colorectal neoplasms. Liver neoplasms. Prognosis.

\section{REFERÊNCIAS}

1. GORDON, P. H. - Principles and practice of surgery for the colon, rectum and anus. 2a. ed., 1999.

2. HUGHES, K.S.; ROSENSTEIN, R.B.; SONGHORABODI, S. - Resection of the liver for colorectal carcinoma metastases: A multi-institutional study of long-term survivors. Dis. Colon Rectum, 1988; 31:1-4).
3. LEE E.T. Statistical methods for survival data analysis. California, Lifetime Learning Publications, p.75-130, 1980.

4. RAWET V. Carcinoma colorretal: estadiamento e parâmetros prognósticos. São Paulo, 1998. 194p. Tese de Doutorado - Faculdade de Medicina, Universidade de São Paulo.

Conflito de interesse: não há

Fonte financiadora: não há

Recebido para publicação em: 08/05/2007

Aceito para publicação em: 21/08/2007 